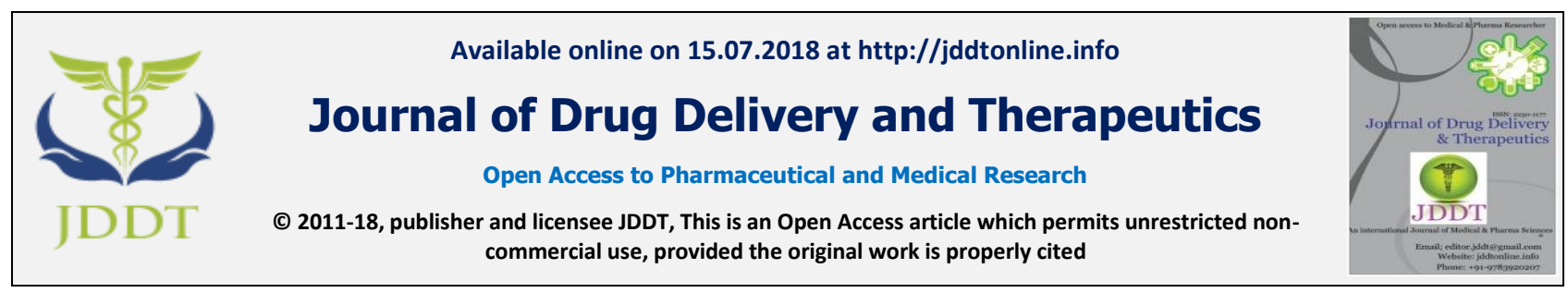

Open $\odot$ Access

Research Article

\title{
STUDY OF SWELLING BEHAVIOR AND DETERMINATION OF SWELLING PARAMETERS OF SPHERICAL HYDROGELS IN WATER
}

\author{
Ashtaputrey Prashant*, Ashtaputrey Santosh \\ Department of Chemistry, Institute of Science, Nagpur, MS, India, 440001
}

\section{ABSTRACT}

Spherical Hydrogels are having the tendency to absorb the large amount of water. This results in swelling of the hydrogels that leads to the increase in mass and volume of hydrogels. In the present work this change in the mass and volume of spherical hydrogels at different time interval has been studied. The different swelling related parameters like mass swelling ratio (MSR), equilibrium swelling ratio (ESR), swelling constant (K) which is the characteristic of polymer network of hydrogel bead, diffusional exponent (n) characterizing the mechanism of diffusion of the solvent into the network , equilibrium water content (EWC), diffusion coefficient of water (D), number of absorbing sites per unit mass of dry hydrogel $\left(\rho_{M}\right)$ and number of absorbing sites per unit volume of dry hydrogel $\left(\rho_{N}\right)$ were determined for four different colored (Red, Blue, Orange and Green) spherical hydrogels. The linear relationship of $\log \left(\frac{Q_{t}}{Q_{e}}\right)$ is observed with $\log (t)$ and also between change in volume and change in mass of hydrogel. The water intake and volume of hydrogel increases with time initially and later there is no appreciable change in water intake and volume due to maximum water absorption i.e. equilibrium swelling of hydrogels.

Keywords: Spherical Hydrogels, Swelling ratio, parameter, water

Article Info: Received 28 April, 2018; Review Completed 22 June 2018; Accepted 27 June 2018; Available online 15 July 2018

Cite this article as:

Ashtaputrey P, Ashtaputrey S, Study of swelling behavior and determination of swelling parameters of spherical hydrogels in water, Journal of Drug Delivery and Therapeutics. 2018; 8(4):218-225 DOI: http://dx.doi.org/10.22270/iddt.v8i4.1775

*Address for Correspondence:

Ashtaputrey Prashant, Department of Chemistry, Institute of Science, Nagpur, MS, India, 440001

\section{INTRODUCTION}

Hydrogels are the three dimensional lightly crosslinked hydrophilic natural or synthetic polymers that can absorb and retain water up to hundreds of times of their own weight and undergo swelling without dissolving in water ${ }^{1}$. Water-soluble polymers such as poly (acrylic acid), poly (vinyl alcohol), poly (vinylpyrrolidone), poly (ethylene glycol), polyacrylamide and some polysaccharides are the most common systems used to form hydrogels. When a dry swellable hydrogel comes in contact with water, a swelling process starts. The polymer absorbs water and increases its volume. Hydrogels are generally characterized by their ultimate capacity to absorb water (swelling thermodynamics), the rate at which the water is absorbed into their structure (swelling kinetics), as well as their mechanical property in wet or hydrated state (wet strength).
The characteristic properties such as expansion in water (swelling), high water content and elastic nature similar to natural tissues, biocompatibility and lack of toxicity, have enabled them to find extensive applications in biomedical ${ }^{2}$, bioengineering and biotechnological ${ }^{3}$, pharmaceutical $^{4}$ areas. The hydrogels are also found wide applications for use as sensors, actuators ${ }^{5}$ etc. Desirable hydrogel properties for a given application can be achieved by selecting a proper hydrogel material, crosslinking method, as well as processing techniques.

Many researchers have already reported the preparation of hydrogels and their swelling behavior in various solvents under different conditions. M. F. Mina et.al ${ }^{6}$ has studied the swelling behavior of acrylamide hydrogel in different solvents at different $\mathrm{P}^{\mathrm{H}}$. M Sivanantham et.al ${ }^{7}$ has investigated the swelling kinetics of water-swollen polyacrylamide hydrogels in various 
concentrations of aqueous sodium chloride solution by macroscopic swelling measurement. Sudipto Roy et.al ${ }^{8}$ has studied the swelling behavior of spherical water gels in Sugar Solution. H.B. Bohidar et.al ${ }^{9}$ have studied the Swelling and de-swelling kinetics of gelatin hydrogels in ethanol-water marginal solvent. Young Chang Nho et.al ${ }^{10}$ has studied the swelling behavior study of $\gamma$ irradiated gelatin hydrogels prepared in organic/aqueous mixtures. Omer Barıs Uzum et.al ${ }^{11}$ have worked on the equilibrium swelling studies of chemically cross-linked highly swollen acrylamide-sodium acrylate hydrogels in various water-solvent mixtures. Priyadarshi Majumdar et.al ${ }^{12}$ has studied the swelling properties of spherical hydrogel immersed in water and formulates a simple theoretical model. In the present research paper, we have studied the swelling behavior of decorative spherical hydrogels purchased from local market in distilled water at room temperature and various swelling parameters were determined. These non toxic and biodegradable hydrogels are mainly used for domestic decoration and as a water retaining agents for ornamental plants.

\section{Experimental:}

\section{Material:}

Spherical Hydrogel beads: The spherical hydrogel beads required for the present study were purchased from the local market. The main composition of these hydrogels is polyacrylate or polyacrylamide. These hydrogels are prepared by the polymerization of acrylic acid blended with sodium hydroxide in the presence of an initiator to form a poly-acrylic acid sodium salt. The purchased hydrogels were of different colours. Out of which red, blue, orange and green coloured hydrogels of uniform spherical shape were selected for the study.

Screw Gauge: The initial diameter of the dry hydrogel and that of swelled hydrated hydrogel at different time interval were measured by making the use of screw guage having least count of $0.01 \mathrm{~mm}$.

Weighing balance: The initial weight of the dry hydrogel and that of hydrated hydrogel at different time interval were measured by making the use of systronics digital electronic balance having accuracy of $0.1 \mathrm{mg}$.

Stop watch: The regular time interval of twenty minutes was measured with the help of stop watch having an accuracy of one second.

\section{Method:}

The easiest way to study the swelling behavior of hydrogels is to disperse the spherical hydrogel beads in water and measuring the change in their volume and weight at regular time intervals. In the present work the regular time interval of twenty minutes was taken for measuring the change in volume and weight of hydrogel. After each time interval the hydrogel beads were taken out of distilled water and surface water of hydrogel was removed by rolling the swelled hydrogel smoothly and carefully on blotting paper. The diameter of hydrogel was measured with the help of screw guage and the weight was taken by using the systronics digital electronic balance. The procedure was repeated until the equilibrium of swelling is reached where the hydrogel bead attained the maximum constant diameter and weight. This method is known as continuous variation method. The various swelling parameters ${ }^{13,14,15}$ were then calculated as given below,

The mass swelling ratio (MSR) denoted by $Q_{t}$ was calculated by the following equation

$$
Q_{t}(M S R)=\frac{M_{t}-M_{0}}{M_{0}}
$$

Where $\quad M_{t}=$ Mass of hydrogel at a particular time $t$.

$$
M_{0}=\text { Initial mass of hydrogel in its dry state. }
$$

The other parameters like equilibrium swelling ratio $Q_{e}$ $(E S R)$ and equilibrium water content $(E W C)$ were calculated when the hydrogels attained the maximum weight and diameter i.e. there was no further change in weight and diameter of hydrogel in water with time, by using the following equations,

$$
\begin{aligned}
& Q_{e}(E S R)=\frac{M_{\max }-M_{0}}{M_{0}} \\
& \mathrm{EWC}=\frac{M_{\max }-M_{0}}{M_{\max }}
\end{aligned}
$$

Where $M_{\max }=$ The mass of the hydrogel when it is in perfect equilibrium with the solvent in contact. It is the maximum attainable mass of a hydrogel bead.

Water molecules migrate into the polymer network by the process of diffusion. The swelling properties of hydrogels are often found to obey following mathematical relation ${ }^{16}$,

$$
\frac{Q_{t}}{Q_{e}}=K t^{n}
$$

iv

Where $\mathrm{K}=$ Swelling constant, characteristic of polymer network.

$\mathrm{n}=$ The diffusional exponent characterizing the mechanism of diffusion of the solvent into the polymer network.

$\mathrm{t}=$ Time interval of swelling measurement in minutes.

The plot of $\log \left(\frac{Q_{t}}{Q_{e}}\right)$ along Y-axis as a function of $\log (t)$ along $\mathrm{X}$-axis is used to determine the values of $n$ and $K$ from slope and intercept of the straight line respectively.

The diffusion coefficient of water (D) moving through the hydrogel ${ }^{17,} \quad 18$ was calculated by the following equation,

$$
D=\pi R_{0}^{2}\left(\frac{\mathrm{K}}{4}\right)^{1 / \mathrm{n}}
$$

Where $\mathrm{D}=$ Diffusion coefficient of water moving through the hydrogel in $\mathrm{cm}^{2} / \mathrm{min}$.

$\mathrm{R}_{0}=$ Radius of the dry hydrogel in $\mathrm{cm}$.

$\mathrm{K}=$ Swelling constant, characteristic of polymer network as determined above.

$\mathrm{n}=$ Diffusional exponent characterizing the mechanism of diffusion of the solvent into the polymer network as determined above.

Now the total number of water absorbing sites in a hydrogel $(\mathrm{N})$ was determined by the equation, 


$$
N=\frac{M_{\max }-M_{0}}{m}
$$

Where $\mathrm{m}=$ mass of water molecule given by the equation,

$$
m=\frac{\text { Molar mass of water }}{\text { Avagadro's Number }}=\frac{18}{6.023 \times 10^{23}}=2.99146 \times 10^{-23} \mathrm{gm}
$$

Number of sites per unit dry volume of hydrogel will be,

$$
\rho_{N}=\frac{N}{V_{0}}
$$

Where $\mathrm{V}_{0}=$ volume of the dry hydrogel.

The number of sites per unit mass of dry of hydrogel will be

$$
\rho_{M}=\frac{\rho_{N}}{\rho_{0}} \quad \text { Where } \quad \rho_{0}=\frac{M_{0}}{V_{0}}
$$
viii

\section{RESULTS AND DISCUSSION}

Hydrogels absorb large amount of water in the process of swelling and this property leads to the importance of hydrogel in many applications like pharmaceutical, agriculture and in household decorative products. The swelling of hydrogels occurs due to crosslinking of hydrogel molecules in solvent and the extent of swelling changes with the density of hydrogel molecular crosslinking. This change of swelling behavior with respect to crosslinking density can be explained by the interaction of the hydrogel molecules with the solvent molecules according to the classical Flory-Huggins theory ${ }^{19}$. According to this theory, the existing pressure involved due to the interaction between the polymer network and the solvent mainly responsible for the expansion or shrinking of the hydrogel. Figure: 1 shows the linear increase in the $\log \left(\frac{Q_{t}}{Q_{e}}\right)$ with $\log (t)$ as expected. The values of $\mathrm{n}$ and $\mathrm{k}$ were obtained from slope and intercept of the same plot respectively and are tabulated in Table 1 . The values of $n$ clearly indicate that relaxation-controlled diffusion process occurs in all four samples.

Increase of swelling with time is an inherent property of hydrogel because of its hydrophilic character. The rate of swelling is more in the beginning and it gradually decreases with time till it attained the equilibrium stage where there is no further swelling of hydrogels due to maximum water absorption. Figure: 2 show the volume of hydrogel at different time interval. From the figure it is seen that initially there is increase in volume with time and later there is no appreciable volume change due to maximum water absorption i.e. equilibrium swelling ratio of hydrogels. Figure: 3 show the linear variation of change of volume of hydrogel with the change in the mass of hydrogel. Figure: 4 show the water intake variation of hydrogel with time. The water intake increases up to certain time and then it remains almost constant due to equilibrium water content. The time period during which the volume change of hydrogel is faster could be utilized in many cases like drug delivery, separation processes and so on. Analysis of the mechanisms of water diffusion into swellable polymeric systems has received considerable interest in recent years, because of important applications of swellable polymers in biomedical, pharmaceutical, environmental and agricultural engineering.

The values of ESR, EWC, Diffusion coefficient (D), Number of active sites $(\mathrm{N})$, Number of sites per unit dry volume of hydrogel $\left(\rho_{\mathrm{N}}\right)$, Number of sites per unit mass of dry of hydrogel $\left(\rho_{M}\right)$ for each sample were calculated by using respective equations and are also given in table 1. It is an important experimental observation that the values of EWC are found to be almost same for each hydrogel sample.

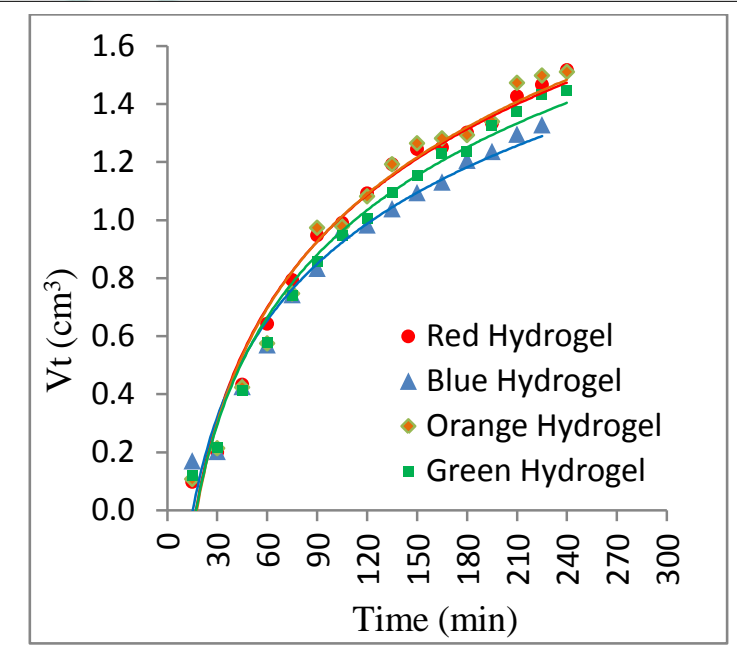

Figure 2: Plot of hydrogel volume $\left(\mathrm{V}_{\mathrm{t}}\right)$ as a function of the time. 


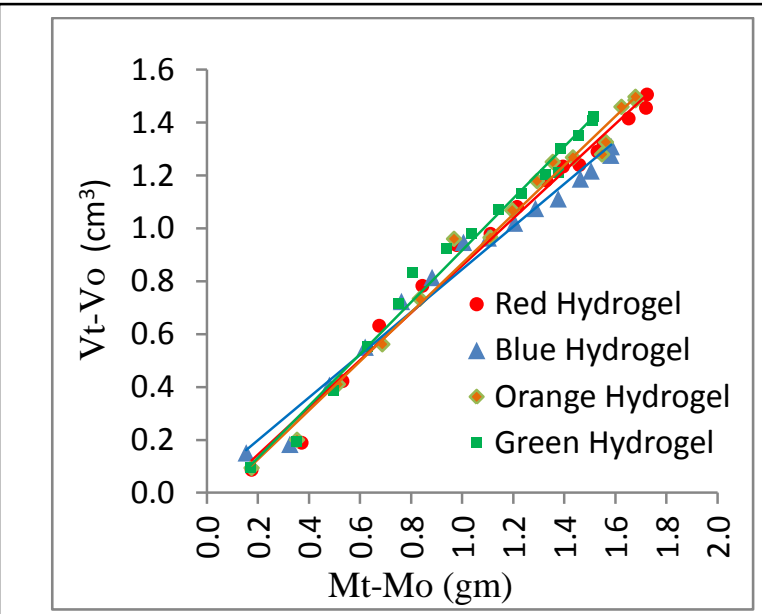

Figure 3: Plots of change in volume of hydrogel $\left(\mathrm{V}_{\mathrm{t}^{-}}\right.$ $\left.\mathrm{V}_{0}\right)$ as a function of the change in mass hydrogel $\left(\mathrm{M}_{\mathrm{t}^{-}}\right.$ $\mathrm{M}_{0}$ )

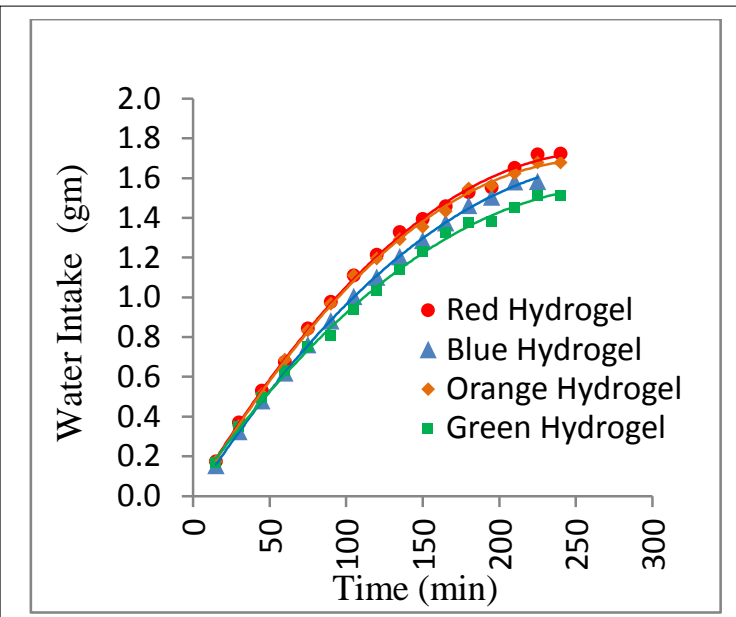

Figure 4: Plot of Mass of water absorbed by hydrogel i.e. water intake as a function of time.

Table 1: Various swelling parameters of hydrogels

\begin{tabular}{|l|c|c|c|c|c|c|c|c|c|c|c|}
\hline Sample & $\begin{array}{c}\mathrm{M}_{0} \\
(\mathrm{gm})\end{array}$ & $\begin{array}{c}\mathrm{M}_{\mathrm{Max}} \\
(\mathrm{gm})\end{array}$ & $\mathrm{K}$ & $\mathrm{n}$ & $\mathrm{ESR}$ & $\mathrm{EWC}$ & $\begin{array}{c}\mathrm{D} \\
\left(\mathrm{cm}^{2} / \mathrm{min}\right)\end{array}$ & $\mathrm{N}$ & $\begin{array}{c}\rho_{0} \\
\left(\mathrm{gm}^{3} \mathrm{~cm}^{3}\right)\end{array}$ & $\begin{array}{c}\rho_{\mathrm{N}^{-3}} \\
\left(\mathrm{~cm}^{-3}\right)\end{array}$ & $\begin{array}{c}\rho_{\mathrm{M}} \\
\left(\mathrm{gm}^{-1}\right)\end{array}$ \\
\hline Red & 0.023 & 1.747 & 1.8644 & 0.8077 & 74.957 & 0.987 & $5.14 \times 10^{-5}$ & $5.7630 \times 10^{22}$ & 2.1616 & $5.4164 \mathrm{X} 10^{24}$ & $2.5056 \mathrm{X} 10^{24}$ \\
\hline Blue & 0.034 & 1.618 & 1.9491 & 0.8521 & 46.588 & 0.979 & $8.74 \times 10^{-5}$ & $5.2950 \times 10^{22}$ & 1.7745 & $2.7636 \times 10^{24}$ & $1.5573 \times 10^{24}$ \\
\hline Orange & 0.032 & 1.545 & 1.6536 & 0.8089 & 47.218 & 0.979 & $7.89 \times 10^{-5}$ & $5.0577 \times 10^{22}$ & 1.3913 & $2.1990 \times 10^{24}$ & $1.5805 \times 10^{24}$ \\
\hline Green & 0.022 & 1.701 & 1.7874 & 0.7726 & 76.318 & 0.987 & $10.90 \times 10^{-5}$ & $5.6126 \mathrm{X} 10^{22}$ & 1.6923 & $5.3174 \mathrm{X} 10^{24}$ & $2.5512 \mathrm{X} 10^{24}$ \\
\hline
\end{tabular}

\section{CONCLUSION}

Outcome of present research work can be explained by considering the interactions among hydrogel polymer molecules and water molecules. The permeability character of water molecules into the crosslinking network of hydrogel polymer plays the major part in their swelling. All four studied hydrogels were found to have swelling parameters very close to one another being they are of same polymeric material polyamide/polyacrylamide. The little differences in the various swelling parameters might be due to added colouring agent. Further there is scope for studying the swelling behavior of hydrogels in different environment like solvents, salt solution and at different $\mathrm{P}^{\mathrm{H}}$.

\section{Acknowledgements:}

The authors gratefully acknowledge the Director, Institute of Science, Nagpur and Head, Department of Chemistry, Institute of Science, Nagpur for providing the facilities and support.

\section{Conflict of Interest:}

The Authors also declare that they have no personal or financial relationships that could be viewed as potential conflict of interest in relation to the publication of present research work. 


\section{REFERENCES}

1. Rosiak JM, Yoshii F, Hydrogels and their medical applications, Nuclear Instruments and Methods in Physics Research B, 1999; 151:56-64.

2. Calo E, Khutoryanskiy VV, Biomedical applications of hydrogels: A review of patents and commercial products, European Polymer Journal, 2015; 65:252-267.

3. Zhang XZ, Yang YY, Chung TS, Effect of mixed solvents on characteristics of poly(N-isopropylacrylamide) gels, Langmuir, 2002; 18:2538-2542.

4. Kashyap N, Kumar N, Kumar MN, Ravi V, Hydrogels for pharmaceutical and biomedical applications, Critical reviews in therapeutic drug carrier systems, 2005; 22(2):107-149.

5. Deligkaris K, Tadele TS, Olthuis W, Van Den Berg A, Hydrogel-based devices for biomedical applications, Sensors and Actuators B: Chemical, 2010; 147(2):765-774.

6. Mina MF, Alam MM, Swelling behavior of acrylamide hydrogel in different solvents and $\mathrm{p}^{\mathrm{H}}$, Chinese Journal of Polymer Science, 2005; 23(3):269-274.

7. Sivanantham M, Tata BVR, Swelling/deswelling of polyacrylamide gels in aqueous $\mathrm{NaCl}$ solution: Light scattering and macroscopic swelling study, Pramanac Indian Academy of Sciences, 2012; 79(3):457-469.

8. Roy S, Majumdar P, Study of the swelling behavior of Spherical water gels in Sugar Solution , International Journal of Drug Development \& Research, 2013; 5(1):252-257.

9. Boral S, Gupta A, Bohidar HB, Swelling and de-swelling kinetics of gelatin hydrogels in ethanol-water marginal solvent, International Journal of Biological Macromolecules 2006; 39:240-249

10. Shin J, Lim YM, Jeun JP, Nho YC, Swelling Behavior Study of $\gamma$-Irradiated Gelatin Hydrogels Prepared in Organic/Aqueous Mixtures, Journal of Industrial and. Engineering Chemistry, 2007; 13(6):997-1001

11. Uzum OB, Karadag E , Equilibrium Swelling Studies of Chemically Cross-Linked Highly Swollen Acrylamide-
Sodium Acrylate Hydrogels in Various Water-Solvent Mixtures, Polymer-Plastics Technology and Engineering, 2010; 49:6:609-616

12. Das P, Biswas D, Roy S, Majumdar P, An experimental study of the swelling properties of spherical hydro-gel immersed in water and the formulation of a simple theoretical model for its explanation, International Journal of Drug Delivery, 2012; 4:20-30.

13. Barati A, Norouzi H, Sharafoddinzadeh S, Davarnejad R, Swelling Kinetics Modeling of Cationic MethacrylamideBased Hydrogels, World Applied Sciences Journal, 2010; 11(11):1336-1341.

14. Karadag E, Saraydin D, Swelling of superabsorbent acrylamide sodium acrylate hydrogels prepared by using multi functional cross-linkers, Turk. J. Chem. 2002; 26:863875.

15. Kim SJ, Park SJ, Kim SJ, Synthesis and characterization of interpenetrating polymer network hydrogels composed of poly (vinyl alcohol) and poly (N-isopropylacrylamide), React. Funct. Polym. 2003; 55:61-67.

16. Saraydın D, Karadag E, Isikver Y, Ahiner S, Guven O, The influence of preparation methods on the swelling and network propertiesof acrylamide hydrogels with crosslinkers, Journal of Macromolecular Science, 2004; A41:419431.

17. Caykara T, Kiper S, Demirel G, Thermosensitive poly (Nisopropylacrylamide-co-acrylamide) hydro-gels: Synthesis, swelling and interaction with ionic surfactants, European Polymer Journal, 2006; 42:348.

18. Korsmeyer RW, Peppas NA, Controlled release delivery systems, Marcel Dekker New York; 1983, P.77.

19. Flory PJ, Principals of Polymer Chemistry, Cornell University, New York; 1953, P.576. 